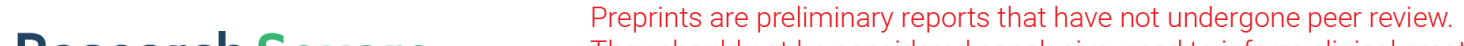 \\ They should not be considered conclusive, used to inform clinical practice, or referenced by the media as validated information. \\ Perspectives of Residency Training Amid The COVID-19 Pandemic: Impact on Mental Health and Training
}

\section{Ashraf Moini}

Tehran University of Medical Sciences

Khadijeh Maajani

Tehran University of Medical Sciences

Ramesh Omranipour

Tehran University of Medical Sciences

Mohammad Reza Zafarghandi

Tehran University of Medical Sciences

Ashraf Aleyasin

Tehran University of Medical Sciences

\section{Roya Oskoie}

Tehran University of Medical Sciences

Sadaf Alipour ( $\nabla$ sadafalipour@yahoo.com )

Tehran University of Medical Sciences

\section{Research Article}

Keywords: Coronaphobia, General Surgery, Gynecology, Mental Health, Obstetrics, Residency, Stress, Training

Posted Date: January 29th, 2021

DOI: https://doi.org/10.21203/rs.3.rs-148405/v1

License: (c) (1) This work is licensed under a Creative Commons Attribution 4.0 International License. Read Full License 


\section{Abstract \\ Background}

COVID-19 has affected the training programs and the clinical schedules of surgical wards in many countries, including Iran. Also, the continuous involvement with COVID-19 patients has caused stress in health care workers; among them residents are on the frontlines of care delivery. Therefore, we designed a study to assess mental effects of these circumstances, and the effects on General Surgery and Obstetrics \& Gynecology residency training.

\section{Methods}

We used a questionnaire consisting of 47 questions about personal, familial and demographic characteristics; training activities, and mental effects of COVID-19.

\section{Results}

The answer rate was $63.5 \%$. (127 filled questionnaires). Around $96 \%$ of the residents had emotional problems, $85.9 \%$ were highly stressed about contracting COVID-19, 81.3\% were worried about transferring it to their family; and $78 \%$ believed that their residency training had been impaired.

\section{Conclusion}

Overall, our study shows the negative impact of COVID-19 on the mental health and on the training of residents. We propose that appropriate emotional support and suitable planning for compensation of training deficits is provided for residents.

\section{Introduction}

COVID-19 conditions have affected people emotionally all over the world; emotional consequences derived from these circumstances have been termed as "Coronaphobia" [1]. Health care workers continuous challenge in the frontline of the anti-COVID battle put them at higher risk [2]. A systematic review by Muller et al [3] about health care workers during the pandemic showed sleep disturbance, depression, anxiety, and distress.

Considering the direct and first line contact of residents with COVID-19 patients, the situation could impose emotional disruptions on them. Therefore, we carried out a study to investigate the effects of dealing with COVID-19 cases on the mental health of the residents of General Surgery (GS) and Obstetrics and Gynecology (OG) in Tehran University of Medical Sciences (TUMS), and the impact on their work and education as perceived by the residents. 


\section{Materials And Methods}

The study protocol was approved by the Deputy of research of TUMS (Code 99-2-100-48539), and the ethical approval was issued by the Ethics Committee of TUMS (Code: IR.TUMS.VCR.REC.1399.499). All methods were carried out in accordance with relevant guidelines and regulations.

A researcher- made questionnaire was handed to all the residents of GS and OG of TUMS. The questionnaire included 15 questions about personal, familial and demographic characteristics; 10 questions about the conditions of the residency activities and training as well as the provided hygienic facilities; 10 questions about the mental effects of COVID-19 based on the validated questionnaire of Khodabakhshi et al [4] and the study of Abdessater et al [5]; and 12 questions from the short General Health Questionnaire (GHQ-12) which has previously been validated in Iran [6, 7]. Residents were requested to answer the last 12 questions by considering the changes they felt in each item due to the COVID-19 conditions. According to the standard GHQ-12, these consisted of 6 positive questions, for example enjoying daily activities; and 6 negative questions, like feeling sad or depressed. We used the 4point Likert scale to rate the answers. In positive questions, the $a, b, c$ and $d$ choices in the answers were rated $3,2,1$, and 0 , respectively. In negative answers the sequence was reversed, so that choice a got zero and choice $d$ was scored as 3 . Therefore, the highest score would be 36. According to Yaghubi et al [7], a cut-off point of 15 was defined (maximum specificity score of $95.8 \%$ ), where scores less than 15 were considered healthy, and scores above 15 indicated disturbed COVID-related mental health status (CMH).

We used mean \pm standard deviation (SD) to describe quantitative variables, and frequency (percent) to describe qualitative variables. The Chi-square test was used for assessment of the association of quantitative variables with $\mathrm{CMH}$, and logistic regression was used to find variables that affected $\mathrm{CMH}$. The significance level was considered $p<0.05$ for all analyzes. All statistical tests were performed by SPSS 26.

\section{Results}

Among the 200 distributed questionnaires, 127 were recruited; showing an answer rate of around $63.5 \%$. Table 1 shows the demographic and personal features, past history and COVID-19 history of the residents. 
Table 1

Demographic and personal characteristics, past history and COVID-19 history of all residents

\section{Variable}

Age (mean \pm SD)

$30.9 \pm 2.4$

Sex

Male

$49(51.6 \%)$

Female

$46(48.4 \%)$

$\mathrm{BMI}($ mean $\pm \mathrm{SD})$

$24.8 \pm 3.4$

Underlying disease*

No

$79 \pm 83.2$

Yes

$16 \pm 16.8$

Underlying disease* in family members**

No

$36 \pm 37.9$

Yes

$59 \pm 62.1$

Marital status

Single

$47(49.5 \%)$

Married

$48(50.5 \%)$

Residency year

First year

$22(22.1 \%)$

Second year

$20(21.1 \%)$

Third year

$28(29.5 \%)$

Fourth year

$25(26.3 \%)$

Smoking

Yes

$12(12.6 \%)$

No

$81(85.3 \%)$

History of COVID-19 affection

No

$61(64.2 \%)$

Yes, based on symptoms

$25(26.3 \%)$

Yes, based on a positive test

$7(7.4 \%)$

Having children

No

$70(73.7 \%)$

Yes

25 (26.3)

Living wih parents

No

69 (72.6)

Yes

24 (25.3)

*Including diabetes mellitus, hypertension, ischemic heart disease or heart failure, respiratory disorders, immunosuppressive states, psychological disorders; ** Including only members of the family who live with the resident; $S D$ = standard deviation 
Regarding educational issues, $62(51.2 \%)$ residents reported a significant decrease in the number of training operations. The COVID-19 pandemic has caused new arrangements in the work hours of professors in order to avoid crowding of hospitals and provide possibility for COVID-19 wards to be visited by specialties for their surgical complaints. Therefore, we inquired residents about any change in the rate of supervision of their professors over their surgical activities in the COVID-19 era. Among all residents, 84 (70.5\%) believed that the presence of their professors in the operating room had decreased, and $101(85.9 \%)$ stated that their residency training had been impaired.

Residents had to attend to COVID-19 patients in two different ways: first, for prenatal care or management of surgical and gynecological diseases of patients affected by COVID-19, second as a general practitioner in compensation for the shortages in residents and specialists of infectious disease in the management of COVID-19 patients. Results of our study showed that 103 (80.5\%) of the residents had to manage COVID-19 patients which were directly related to their field of residency, and 48 (37.5\%) had to take care of COVID-19 patients that were not related to their field. Seventy-four of the residents $(61.1 \%)$ stated that they had been instructed about the suitable approach to COVID-19 patients, and 87 $(71.9 \%)$ declared that they could appropriately manage these cases. Among them, only $74(61.7 \%)$ stated that appropriate personal protective equipment (PPE) was provided for them by the hospital.

None of the residents had a previous history of mental disorder. However, the questionnaires showed that 123 (96.1\%) experienced emotional problems in the COVID-19 circumstances. Fifty-six residents (45.5\%) reported being highly stressed about the possibility of getting affected by the disease, while 100 (81.3\%) were worried about transferring the virus to their family. In addition, 54 residents (43.9\%) had become excessively obsessed for cleaning and disinfecting themselves and their clothes. The worries of the residents about themselves and their family were not related with their affection with COVID-19 $(p=0.56)$, their underlying disease $(p=0.62)$, smoking history $(p=0.59)$, their presence in COVID ward $(p=0.65)$, the PPE supplied by the hospital $(p=0.87)$, and the physical health status of their family $(p=0.91)$.

Logistic regression analysis did not show any statistically significant association between $\mathrm{CMH}$ and sex $(p=0.4)$, history of affection by COVID-19 $(p=0.69)$, underlying diseases in the resident $(p=0.99)$ or the family members $(p=0.42)$, supplies of PPE $(p=0.99)$, presence of professors $(p=0.7)$, managing COVID19 patients $(p=0.1)$, and practicing in COVID-19 wards $(p=0.1)$.

\section{Discussion}

We performed a study to investigate the conditions of residency training as well as the CMH of GS and OG residents during the COVID-19 pandemic, and find out a high level of changes in both training and mental health status. To our knowledge, no similar study has been performed about residents in Iran, and studies in other countries have not considered training conditions and mental health status of residents as we did. However, several studies about effects of the pandemic on residency training have been performed in different countries. Brito et al [8] investigated the status of OG residents in Brazil, and found that nearly a third were assigned to activities not related to their field of study, around a quarter 
complained about defective supplies of PPE, and a third had not got instructed about the management of COVID-19 cases. Bitonti et al [9] studied 476 OG residents in Italy and found out that around $6 \%$ had tested positive for COVID-19, only $80 \%$ had been instructed about preventive protocols, and nearly half had been provided with adequate PPE. Aziz et al [10] studied 1102 GS residents in the USA and detected a significant negative impact on surgical training of the residents, who also were worried about contracting the disease by themselves or their family. He et al [11] examined the concerns of GS residents about the pandemic in USA, and detected that they were worried about getting COVID-19, transferring the disease to their family or to the patients. Collins et al [12] surveyed residents of GS and plastic surgery in USA regarding anxiety at time of COVID-19. They found that residents were worried about their training, but also had significant anxiety about their family and self-health. Osama et al [13] assessed the subject in 112 surgery residents of a tertiary care hospital in Pakistan, a high percentage of whom reported impaired surgical training during the pandemic. Their next concern was about their family health, and then fear of death from COVID-19.

Except for the assessment of the worries and anxiety of residents about their health and that of their family, or about defective training, none of these studies examined the mental health status of the residents during COVID-19 by validated methods. However, use of GHQ-12 gave standard results in our study, confirming high levels of stress and emotional affection in the residents.

All the investigations in other countries showed a decrease of training opportunities, which is fairly expected in the COVID-19 era and similar results were driven from ours. Emotional consequences or Coronaphobia was very prevalent in our small population of GS and OG residents.

\section{Conclusion}

Our study shows the negative impact of COVID-19 on the mental health and on the training of residents. We recommend serious planning in order to provide adequate emotional support for residents during the present circumstances, and suitable arrangements by the academic staff to compensate for the current deficit in residency training.

\section{Declarations}

\section{Ethical Considerations}

The study received ethical approval from the Ethics Committee of TUMS (Ethics CODE:

IR.TUMS.VCR.REC.1399.499), and informed consent forms were handed to the residents with the questionnaires and signed by them.

\section{Conflict of Interest}

The authors have no conflict of interest to declare. 


\section{Funding}

This study was not supported by any funding.

\section{Consent for publication}

All the authors consent to the publication of this article in BMC Medical Education.

\section{Availability of data and materials}

Data and materials are in SPSS format and are in possession of the authors; they are available by request from the corresponding author at sadafalipour@yahoo.com.

\section{Authors' contributions}

A.M., R.Om., M.R.Z., A.A., and S.A. designed the study and the questionnaires. A.M., M.R.Z., and A.A. supervised the accuracy of the activities. R.Om. supervised data collection. R.Os. gathered the data. K.M. did the analyses. S.A. and K.M. wrote the main manuscript text. All authors reviewed the manuscript.

\section{Acknowledgements}

We would like to acknowledge Mr. Hamid Razavian, Mrs. Marzieh Orouji, Mrs Khorshid Mansouri and Mrs, Hamideh Shahriari for their active collaboration in data gathering.

\section{References}

1. Arora A, Jha AK, Alat P, Das SS: Understanding coronaphobia. Asian Journal of Psychiatry 2020:102384.

2. Amin S: The psychology of coronavirus fear: Are healthcare professionals suffering from coronaphobia? International Journal of Healthcare Management 2020:1-8.

3. Muller RAE, Stensland RS $\emptyset$, van de Velde RS: The mental health impact of the covid-19 pandemic on healthcare workers, and interventions to help them: a rapid systematic review. Psychiatry Research 2020:113441.

4. Khodabakhshi-koolaee A: Living in home quarantine: Analyzing psychological experiences of college students during COVID-19 pandemic. Journal of Military Medicine 2020, 22(2):130-138.

5. Abdessater M, Rouprêt M, Misrai V, Matillon X, Tellier BG, Freton L, Vallée M, Dominique I, Felber M, Khene Z-E: COVID19 pandemic impact on anxiety of French urologists in training: outcomes from a national survey. Progres En Urologie 2020.

6. Montazeri A, Harirchi AM, Shariati M, Garmaroudi G, Ebadi M, Fateh A: The 12-item General Health Questionnaire (GHQ-12): translation and validation study of the Iranian version. Health and quality of life outcomes 2003, 1(1):66. 
7. Yaghubi H, Karimi M, Omidi A, Barouti E, Abedi M: Validity and factor structure of the General Health Questionnaire (GHQ-12) in university students. J Behav Sci 2012; 6 (2): 153-160. common mental disorders. Soc Sci Med 2008, 66(4):1008-1018.

8. Brito LG, Romão GS, Fernandes CE, Silva-Filho AL: Impact of COVID-19 on Brazilian medical residencies in obstetrics and gynecology. International Journal of Gynecology \& Obstetrics 2020 , 150(3):411-412.

9. Bitonti G, Palumbo AR, Gallo C, Rania E, Saccone G, De Vivo V, Zullo F, Di Carlo C, Venturella R: Being an obstetrics and gynaecology resident during the COVID-19: Impact of the pandemic on the residency training program. European Journal of Obstetrics \& Gynecology and Reproductive Biology 2020, 253:48-51.

10. Aziz H, James T, Remulla D, Sher L, Genyk Y, Sullivan ME, Sheikh MR: Effect of COVID-19 on surgical training across the United States: a national survey of general Surgery residents. Journal of Surgical Education 2020.

11. He K, Stolarski A, Whang E, Kristo G: Addressing General Surgery residents' concerns in the early phase of the COVID-19 pandemic. Journal of Surgical Education 2020.

12. Collins $C$, Mahuron K, Bongiovanni T, Lancaster E, Sosa JA, Wick E: Stress and the Surgical Resident in the COVID-19 Pandemic. Journal of surgical education 2020.

13. Osama M, Zaheer F, Saeed H, Anees K, Jawed Q, Syed SH, Sheikh BA: Impact of COVID-19 on surgical residency programs in Pakistan; A residents' perspective. Do programs need formal restructuring to adjust with the" new normal"? A cross-sectional survey study. International Journal of Surgery 2020. 